\title{
A case of ulceroglandular tularemia presenting with lymphadenopathy and an ulcer on a linear morphoea lesion surrounded by erysipelas
}

This article was published in the following Dove Press journal: International Medical Case Reports Journal

\author{
Alessandra Balestra' \\ Hekuran Bytyci' \\ Caroline Guillod ${ }^{2}$ \\ Antonio Braghetti ${ }^{3}$ \\ Luigia Elzi ${ }^{4}$
}

'Department of Internal Medicine, Regional Hospital of Bellinzona e Valli, 6500 Bellinzona, Ticino, Switzerland;

2Department of Dermatology, Regional Hospital of Bellinzona e Valli, 6500 Bellinzona, Ticino, Switzerland; ${ }^{3}$ Department of Radiology, Regional Hospital of Bellinzona e Valli, 6500 Bellinzona, Ticino, Switzerland; ${ }^{4}$ Department of Infectious Diseases, Regional Hospital of Bellinzona e Valli, 6500 Bellinzona, Ticino, Switzerland

Correspondence: Alessandra Balestra Department of Internal Medicine, Regional Hospital of Bellinzona e Valli, Corzoneso Piano 6, 6722 Acquarossa, Bellinzona, Ticino, Switzerland

$\mathrm{Tel}+4 \mid$ 9| 8 II 2522

Fax +41918112517

Email alessandra.balestra@eoc.ch

\begin{abstract}
Tularemia is a zoonosis caused by the infection of Francisella tularensis (a gramnegative aerobic bacterium). Transmission to other animals or humans usually occurs through insect or tick bites, direct contact with a contaminated environment (mud or water), infected animals - mainly lagomorphs - or by ingesting undercooked meat or inhaling contaminated dust (hay or soil). This paper discusses the case of a 32-year-old man, who came to our Emergency Room presenting with persistent fever, inguinal lymphadenopathy, and an ulcer on his left lower limb on a linear morphoea lesion that had been there for some time. The lesion was surrounded by erysipelas. After hospitalization and tests, the patient was diagnosed with ulceroglandular tularemia. Antibiotic treatment with doxycycline resolved the clinical picture, but not the morphoea lesion.
\end{abstract}

Keywords: tularemia, Francisella tularensis, zoonosis, erysipelas, lagomorphs

\section{Introduction}

Francisella tularensis (F. tularensis) are transmitted to other animals or humans through insect or tick bites, direct contact with a contaminated environment, infected animals, or by ingesting undercooked meat or inhaling contaminated dust. ${ }^{1,2}$ Ulceroglandular tularemia, one of the six main clinical forms of this disease, is the most common and clinically recognizable. Symptoms include fever, inflammation of the infected area, and enlarged lymph nodes. ${ }^{9}$ Treatment include gentamicin intramuscular or intravenous for moderate to severe infections and doxycycline or ciprofloxacin for milder infections. ${ }^{13}$

\section{Case report \\ Medical history}

A 32-year-old man presenting with morphoea (circumscribed scleroderma) on his left lower limb had been regularly treated with topical steroids by his dermatologist. The patient also presented with recurrent ulcers on the lower limbs, which had been evaluated on several occasions at a hospital in Zurich and attributed to microtraumas. However, the patient did not report any trauma during history-taking. He was not taking medications and did not have any known allergies. He did report to have regular contact with his cat and dog but did not remember to have ever been bitten. He did not remember any tick bites either, and he had not had the opportunity to travel abroad over the previous 8 weeks. He is a carpenter.

The patient came to the Emergency Room of our Regional Hospital presenting with left groin pain and a body temperature of up to $39^{\circ} \mathrm{C}$ with shivers. Blood tests showed 
leukocytosis (14 g/L) with normal C-reactive protein (CRP). An inguinal ultrasound examination showed subcutaneous edema and numerous benign reactive enlarged lymph nodes (Figure 1).

A chest X-ray did not reveal any suspicious alterations. The images of the pelvic X-ray were compatible with trochanteric insertional tendinopathy. Therefore, the patient was diagnosed with acute pubalgia in insertional tendinopathy and discharged with anti-inflammatory treatment.

However, the next day, the patient returned to the Emergency Room as the groin pain had gotten worse.

\section{Status}

The patient's vitals were characterized by high body temperature $\left(39.2^{\circ} \mathrm{C}\right)$, hypotension $(106 / 46 \mathrm{mmHg})$, tachycardia (116 bpm), and normal saturation. The lower left limb had a $12 \times 6 \mathrm{~mm}$ superficial pretibial ulcer with a fibrin bed on the (healed) linear morphoea lesion we knew about, and erythema with ill-defined borders (Figure 2). The patient also presented with erythema on the thigh, which extended to the lower limb (Figure 3).

We suspected left leg erysipelas due to secondary infection of the underlying morphoea lesion. The patient was admitted to the Internal Medicine Department, where he was given an intravenous antibiotic treatment with a highdose $(2.2 \mathrm{~g})$ formulation of amoxicillin/clavulanic acid three times a day.

\section{Results}

During the following days the fever persisted, accompanied by the worsening of the localized edema and an increase in inflammatory parameters, with CRP peaking at $329 \mathrm{mg} / \mathrm{L}$ on day 5 and leukocytosis at $15 \times 10^{9} / \mathrm{L}$ on day 7 . The erythrocyte sedimentation rate was $110 \mathrm{~mm} / \mathrm{h}$. Tests also showed a

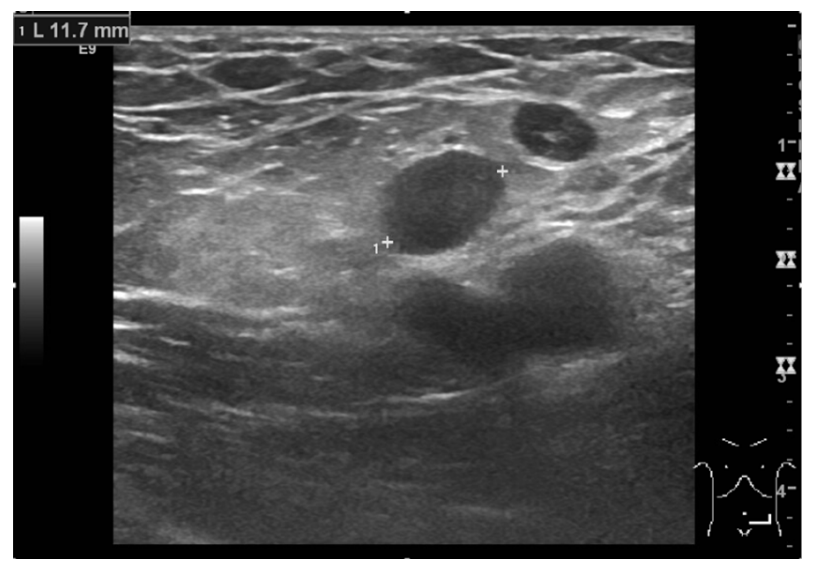

Figure I An inguinal ultrasound examination showed subcutaneous edema and numerous benign reactive enlarged lymph nodes.

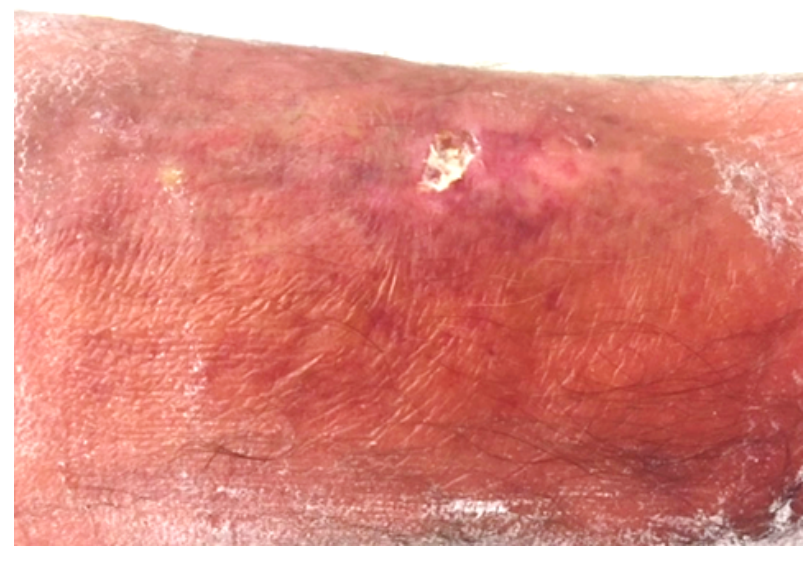

Figure 2 A $12 \times 6 \mathrm{~mm}$ pretibial ulcer with a fibrin bed on the linear morphoea lesion and erythema with ill-defined borders.

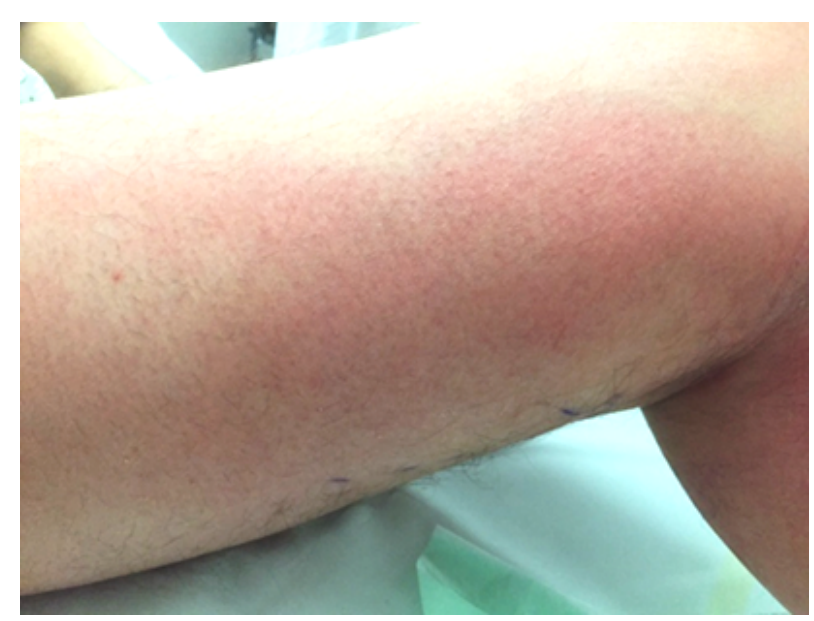

Figure 3 Erythematous lymph nodes on the medial side of the ipsilateral thigh up to the groin.

Note: Two small ulcerated lesions at malleolar level.

positive antistreptolysin titer $(508 \mathrm{U} / \mathrm{L})$. The antibiotics were switched to carbapenems (imipenem/cilastatin $500 \mathrm{mg} / 500$ mg four times a day), which led to a slight improvement, although the fever persisted. The patient was then transferred to the Department of Dermatology for specialist care.

During his stay, four pairs of blood cultures were collected at febrile peaks, but no microorganisms developed from them. The perimalleolar ulcer smears and the HIV serological tests were also negative. The lower extremity venous duplex examination excluded any deep venous thrombosis, while the soft tissue ultrasound of the inguinal region showed reactive subcutaneous lymphadenopathy surrounded by edematous inflammatory tissue with no evidence of abscess. The ankle X-ray showed no findings indicative of osteomyelitis. At that point, our colleagues 
at the Department of Dermatology asked the Department of Infectious Diseases for a consultation. Serological tests for F. tularensis and Bartonella henselae and Bartonella quintana were carried out. Differential diagnosis indicates something intermediate between an infection caused by Francisella, infection by Bartonella (because of the enlarged lymph node), and/or streptococcal infection (positive antistreptolysin titer) starting from the traumatic pretibial ulcer. Our laboratory uses an immunofluorescent assay (IFA) for B. henselae and B. quintana (IFA-IgM and IFA-IgG) and traditional ELISA enzyme immunoassay for F. tularensis, giving results for both IgM ang IgG. At the same time, a doxycycline treatment was administered in addition to the imipenem/cilastatin combination.

\section{Diagnosis and treatment}

The serological test for $B$. quintana and B. henselae was negative (B. quintana IgM: $<1 / 20 ;$ B. quintana IgG: $<1 / 64$; B. henselae IgM: $<1 / 20 ;$ B. henselae IgG: < 1/64) while the serological test for $F$. tularensis was positive (IgM: $33.4 \mathrm{U} /$ $\mathrm{mL}$; IgG: $3 \mathrm{U} / \mathrm{ml}$ ). Therefore, the patient was diagnosed with ulceroglandular tularemia with leg erysipelas secondary to the underlying morphoea lesion. The carbapenem treatment was suspended, so the patient was now treated only with doxycycline. Dalibour water compresses with cream and a silver sulfadiazine pack were applied locally. A class III corticosteroid was applied to the perilesional erythematous skin. Finally, a compression bandage was applied to the patient's left leg.

\section{Evolution}

From a clinical point of view, the patient showed a rapid remission of symptoms. The fever disappeared even after the first dose of antibiotic, and the lymphadenopathy regressed gradually. Upon discharge, CRP levels had decreased to 25 $\mathrm{mg} / \mathrm{L}$ without leukocytosis. The patient continued the antibiotic treatment for 3 weeks.

The Cantonal Medical Service has requested an environmental investigation to identify the source of infection, which is still unknown.

\section{Discussion}

Tularemia is a zoonosis caused by F. tularensis, a gram-negative aerobic bacterium. Subspecies tularensis is the most virulent strain, whereas subspecies holarctica causes most of the infections in humans and animals. This microorganism mainly infects lagomorphs, ie, rabbits, hares, rodents, beavers, muskrats, squirrels, hamsters, and voles. F. tularensis can persist for several weeks in animal carcasses, mud, and water. Laboratory personnel, farmers, veterinarians, hunters, landscapers, and butchers can come in contact with infection sources at work. Contact with infected animals, cats, birds, insects that bite or sting (especially ticks), and contaminated food and water also pose a threat. This infection is usually transmitted to other animals or humans through insect or tick bites, direct contact with a contaminated environment or infected animals (animal hunting, skinning, or slaughtering), or by ingesting undercooked meat or inhaling contaminated dust (eg, hay or soil). Person-to-person transmission has never been reported. ${ }^{1,2}$

\section{Epidemiology}

Many cases of tularemia have been reported in North America, Japan, Russia, Central Asia, Nordic countries, and the Balkans. Although epidemics occurred also in Russia and Eastern Europe, Nordic countries and the USA remain endemic countries for this disease. The annual incidence of tularemia in the USA is $0.5-5$ cases per million people. In the period between 2001-2010, the incidence of tularemia in Kosovo was 5.2 cases per 100,000 people per year, the highest in Europe. ${ }^{3,4}$ Interestingly, tularemia has not been reported in the UK, Iceland, Africa, South America, and Antarctica. Until 2014 Australia has reported only two cases of tularemia related to F. tularensis. ${ }^{5,6}$ Over the past few years, climate change, wars, natural disasters, human travels, and animal migration have been the main causes of the increasing number of tularemia cases worldwide. Global warming has caused an increase in the average temperature throughout the year, which has led to a significant change in the cycle of tularemia. A study carried out in Sweden - in which a climate scenario was created with a $2{ }^{\circ} \mathrm{C}$ increase in the annual average temperature for the time period 2010-2100 - has shown an increase in outbreaks of F. tularensis. ${ }^{7}$

In Switzerland (with a population of 8,482,200 at the end of 2017), ${ }^{8}$ where case reporting is mandatory, the average incidence is seven cases per year. Over the past 10 years, the highest number of cases was reported in 2017 with 66 cases.

\section{Pathogenesis}

The incubation period is 3-5 days (more rarely $1-21$ days). The microorganism proliferates in the inoculation site and, after exiting infected cells through lysis, it causes an acute inflammatory reaction that results in tissue necrosis. It then spreads to the regional lymph nodes and throughout the body via the lymphohematogenous route. 


\section{Clinical signs}

The course of the disease varies depending on modes of transmission, affected organs, and pathogenic subspecies. It usually manifests with fever, progressive inflammation of the infected area, and enlarged lymph nodes. Untreated tularemia infections are fatal in $5 \%-15 \%$ of cases. ${ }^{1,2}$

There are six main clinical forms of tularemia based on the inoculation site and virulence of the infectious agent. These are described in the following.

Ulceroglandular tularemia is the most common and clinically recognizable form. Usually, the patient has had some recent contact with animals or has been exposed to vectors (insects, especially ticks). Clinical signs include fever, an erythematous papule with a central eschar where inoculation occurred (eg, tick bite), and frequent regional, cervical, or occipital firm, elastic adenopathies.

Glandular tularemia manifests with painful regional lymphadenopathy involving one or more lymph nodes, without any identifiable skin lesion. This form is transmitted in the same way as the ulceroglandular form but does not have the characteristic skin lesion at the inoculation site. Absent or blunted fever may contribute to diagnostic delays.

The differential diagnosis for both glandular and ulceroglandular tularemia includes cat-scratch disease, neoplasms, syphilis, mycobacterial infection, lymphogranuloma venereum, streptococcal or staphylococcal lymphadenitis, toxoplasmosis, Herpes simplex, fungal infection, anthrax, and the plague., ${ }^{9,10}$

Oropharyngeal tularemia presents with a sore throat (with or without ulceration) and marked cervical lymphadenopathy, which can be unilateral.

Pneumonic tularemia may occur as a primary infection after inhalation of infected aerosols or as secondary infection following hematogenous spread of organisms in other forms of the disease. ${ }^{11}$ Infected pleural fluid is exudative, negative on Gram stain, and usually contains more than 1,000 leukocytes $/ \mathrm{mm}^{3} .{ }^{12}$ Oculoglandular tularemia is characterized by purulent conjunctivitis, palpebral ulcers, and periocular lymphadenopathy. ${ }^{13}$

Typhoidal tularemia manifests without regional lymphadenopathy or other distinctive clinical signs. It is often associated with underlying chronic systemic diseases or even sepsis. ${ }^{10,14}$

\section{Diagnosis}

In clinical practice, the diagnosis of tularemia remains based on serological methods. ${ }^{1}$ However, a recent study to evaluate the performance of the serological tests available, ie, ELISA
(Serion ELISA) and immunochromatographic test (ICT) compared to that of the microscopic agglutination test (MAT) and indirect immunofluorescence assay (IFA), highlighted the limits of the serological methods. The traditional IgG and IgM ELISA tests for F. tularensis are two commercial kits that allow specific detection of IgM or IgG antibodies. ICT and MAT tests detect both anti-Francisella IgM and IgG, while for IFA detection it is necessary to use two separate tests, one for IgM antibodies (IFA-IgM) and one for IgG (IFA-IgG).

The results of the study showed how ELISA performed better than MAT and IFA in detecting F. tularensis antibodies in serum samples taken from patients with tularemia during the first 2 weeks after the onset of symptoms. However, early detection with low F. tularensis antibody titers can be associated with a higher risk of false-positives due to cross-reacting antibodies, especially between Francisella, Brucella, Yersinia enterocolitica species, and mimivirus capsid antigens. The residual antibody titers may persist for years, leading to false-positives. ELISA tests allow for the early detection of specific antibodies but have lower specificity than MAT and IFA and need to be confirmed by these. On the other hand, the ELISA tests available on the market for the detection and titration of $F$. tularensis IgM or IgG antibodies are easier to automate and standardize. ${ }^{15}$

IgM for F. tularensis usually appear after 2-3 weeks from beginning of symptoms, and may persist together with IgG for longer than 10 years after infection. A definitive serological diagnosis should be supported by seroconversion or a 4-fold titer increase, or confirmed by a positive specific PCR of biopsy specimen. ${ }^{12}$ In our report, unfortunately, a convalescent serum of our patient was not available, neither was there a serum sample before symptoms started, therefore diagnosis of tularemia relied on a positive IgM in the presence of a compatible acute clinical picture and a immediate effectiveness of the specific treatment with doxycycline.

As for the ICT test, this study revealed a much lower specificity than MAT, IFA, and ELISA tests. Its use in hospital laboratories should be discouraged; however, it remains a valuable tool for field studies, as it allows for quick and easy screening of people and animals with potential $F$. tularensis infection.

In the case we are describing, the Infectious Diseases Department did not suggest lesion biopsy because F. tularensis is rarely detected in clinical samples by culture or PCR. ${ }^{15}$

\section{Treatment}

The antibiotic treatment must be administered in all suspected or confirmed cases, although there have been cases of 
spontaneous regression without a specific treatment. ${ }^{9}$ Aminoglycosides (gentamicin) are the drugs of choice for severe infections. Tetracyclines (doxycycline) - also widely used for the prophylaxis and treatment of tularemia - are associated with a higher relapse rate. Recent experience has demonstrated the excellent efficacy of ciprofloxacin, which is preferred for oral use. The treatment period is 10-14 days with aminoglycosides and ciprofloxacin and 21 days with tetracyclines. ${ }^{13}$ Suppurative lymph nodes require surgical drainage; pleural effusion requires thoracentesis to rule out empyema. ${ }^{9,10}$

\section{Prevention}

People exposed to the risk of infection can undergo antibiotic prophylaxis under medical supervision to prevent the onset of the disease. ${ }^{1}$

There is no safe, authorized vaccine available on the market. A potential candidate vaccine (LVS - Live Vaccine Strain) does not meet the criteria for general use. The first live attenuated vaccine candidate was made from $F$. tularensis subsp. holarctica strains in the former Soviet Union and, after serial passage in the USA, it led to the preparation of LVS. Although it demonstrated the ability to induce protective immunity against a low-dose aerosol challenge of the Schu $S 4$ strain, the Food and Drug Administration did not approve LVS as a human vaccine due to its undefined attenuation mechanism, insufficient protection against respiratory infection by type A strains of Francisella, phenotypic instability, and partial virulence after aerosol vaccination.

Therefore, although live attenuated vaccines can be promising, for safety reasons, current trends in prophylaxis are focusing on vaccines based on bacterial subunits rather than on live attenuated strains. Subunit vaccines are considered safer due to their composition, which consists of synthetic or isolated microbial antigens. However, since Francisella is an intracellular pathogen, a Francisella subunit vaccine must induce a cell-mediated response, and the identification of specific T-cell epitopes is not a trivial matter. Computational whole-genome analysis, which detects immunogenic Francisella peptides associated with major histocompatibility complex class I (MHCI), constitutes one of the most promising approaches. Alternatively, a protein array based approach could identify major histocompatibility complex class II (MHCII) epitopes of various serological targets. These new approaches are expected to provide new peptide epitopes for the development of effective subunit vaccines. ${ }^{16}$

\section{Essentials for medical practice}

F. tularensis mainly infects lagomorphs, but can persist for several weeks in animal carcasses, mud, and water. The infection is maintained through the life cycles between ticks and rodents.

Tularemia manifests 3-5 days after incubation, depending on how it was transmitted. Symptoms include fever, inflammation of the infected area, and enlarged lymph nodes. Of the six main clinical forms of this disease, ulceroglandular tularemia is the most common and clinically recognizable.

Treatment must be administered in all suspected or confirmed cases. Moderate to severe infections are usually treated with $5 \mathrm{mg} / \mathrm{kg}$ of gentamicin a day administered intramuscularly or intravenously every 8 hours for 10-14 days. Milder infections are treated with $100 \mathrm{mg}$ of doxycycline administered orally twice a day for 21 days or with 500-750 mg of ciprofloxacin administered orally twice a day for 10-14 days.

\section{Disclosure}

The authors report no conflicts of interest in this work.

\section{References}

1. Bundesamt für Gesundheit - Abteilung Übertragbare Krankheiten. Tularämie/Hasenpest. Bern, Switzerland: Bundesamt für Gesundheit. Available from: https://www.bag.admin.ch/bag/de/home/krankheiten/ krankheiten-im-ueberblick/tularaemie.html. Accessed September 29, 2018.

2. Geyer SJ, Burkey A, Chandler FW. Tularemia. In: Connor DH, editor Pathology of Infectious Diseases. Stamford, CT: Appleton \& Lange; 1997:869.

3. Gürcan Şaban. Epidemiology of Tularemia. Department of Medical Microbiology, Trakya University Faculty of Medicine, Edirne, Turkey Balkan Med J. 2014;31(1):3-10.

4. Grunow R, Kalaveshi A, Kühn A, Mulliqi-Osmani G, Ramadani N. Surveillance of tularaemia in Kosovo, 2001 to 2010. Euro Surveill. 2012;17(28):20217.

5. Jackson J, Mcgregor A, Cooley L, et al. Francisella tularensis subspecies holarctica, Tasmania, Australia, 2011. Emerg Infect Dis. 2012;18(9):1484-1486.

6. Whipp MJ, Davis JM, Lum G, et al. Characterization of a novicidalike subspecies of Francisella tularensis isolated in Australia. J Med Microbiol. 2003;52(Pt 9):839-842.

7. Rydén P, Sjöstedt A, Johansson A. Effects of climate change on tularaemia disease activity in Sweden. Glob Health Action. 2009;2:2063-2067.

8. Bundesamt für Statistik - Sektion Demografie und Migration. Die Bevölkerung der Schweiz. Neuchâtel, Switzerland: Bundesamt für Statistik. Available from: https://www.bfs.admin.ch/bfs/de/home/statistiken/bevoelkerung/standentwicklung/bevoelkerung.html. Accessed September 29, 2018.

9. Evans ME, Gregory DW, Schaffner W, Mcgee ZA. Tularemia: a 30-year experience with 88 cases. Medicine. 1985;64(4):251.

10. Penn RL. Francisella tularensis (tularemia). In: Mandell GL, Bennett JE, Dolin R, editors. Mandell, Douglas, and Bennett's Principles and Practice of Infectious Diseases. 7th ed. Philadelphia, PA: Churchill Livingstone; 2010:2590.

11. Bellido-Casado J, Pérez-Castrillón JL, Bachiller-Luque P, et al. Report on five cases of tularaemic pneumonia in a tularaemia outbreak in Spain. European Journal of Clinical Microbiology \& Infectious Diseases. 2000;19(3):218-220.

12. Bennett JE, Dolin R, Martin BMJ. Mandell, Douglas, and Bennett's Principles and Practice of Infectious Diseases. 8th ed. Amsterdam, Netherlands: Elsevier Health Sciences; 2015:2595-2597. 
13. American Public Health Association. Control of Communicable Diseases Manual. 20th ed. Heymann DL, editor. Washington, DC: American Public Health Association; 2015.

14. Lester Rothfeldt LK, Jacobs RF, Wheeler JG, Weinstein S, Haselow DT. Variation in Tularemia Clinical Manifestations-Arkansas, 2009-2013. Open Forum Infect Dis. 2017;4(1):ofx027ofx027.
15. Yanes H, Hennebique A, Pelloux I, et al. Evaluation of In-House and Commercial Serological Tests for Diagnosis of Human Tularemia. J Clin Microbiol. 2018;56(1).

16. Putzova D, Senitkova I, Stulik J. Tularemia vaccines. Folia Microbiol. 2016;61(6):495-504.

\section{Publish your work in this journal}

The International Medical Case Reports Journal is an international, peer-reviewed open-access journal publishing original case reports from all medical specialties. Previously unpublished medical posters are also accepted relating to any area of clinical or preclinical science. Submissions should not normally exceed 2,000 words or
4 published pages including figures, diagrams and references. The manuscript management system is completely online and includes a very quick and fair peer-review system, which is all easy to use. Visit $\mathrm{http}: / / \mathrm{www}$.dovepress.com/testimonials.php to read real quotes from published authors.

Submit your manuscript here: https://www.dovepress.com/international-medical-case-reports-journal-journal 